\title{
First-principles simulations of ionic molecular solids: The phase transitions inK2Se04
}

\section{Citation}

Lu, H. M., and J. R. Hardy. 1990. "First-Principles Simulations of lonic Molecular Solids: The Phase Transitions inK2Se04." Physical Review Letters 64 (6) (February 5): 661-664. doi:10.1103/ physrevlett.64.661.

\section{Published Version}

doi:10.1103/PhysRevLett.64.661

\section{Permanent link}

http://nrs.harvard.edu/urn-3:HUL.InstRepos:32417393

\section{Terms of Use}

This article was downloaded from Harvard University's DASH repository, and is made available under the terms and conditions applicable to Other Posted Material, as set forth at http:// nrs.harvard.edu/urn-3:HUL.InstRepos:dash.current.terms-of-use\#LAA

\section{Share Your Story}

The Harvard community has made this article openly available.

Please share how this access benefits you. Submit a story.

\section{Accessibility}




\title{
First-Principles Simulations of Ionic Molecular Solids: The Phase Transitions in $\mathrm{K}_{2} \mathrm{SeO}_{4}$
}

\author{
H. M. Lu and J. R. Hardy \\ Department of Physics and Center for Electro-Optics, University of Nebraska-Lincoln, Lincoln, Nebraska 68588-0111 \\ (Received 30 August 1989)
}

\begin{abstract}
We present a new approach to first-principles simulations of the statics and dynamics of ionic molecular crystals. It is shown that the new method gives very realistic simulations of the phase transitions in $\mathrm{K}_{2} \mathrm{SeO}_{4}$ and that a double-well type of structure in the potential-energy surface is the driving mechanism of these phase transitions.

PACS numbers: $64.70 . \mathrm{Kb}, 64.70 . \mathrm{Rh}$
\end{abstract}

First-principles simulations of complex ionic solids using $a b$ initio pairwise interionic potentials have been highly successful in explaining melting, ${ }^{1}$ superionicity, ${ }^{2}$ and zone-boundary instabilities in halide perovskites. ${ }^{3,4}$ With the rigid-ion assumption and the short-range potentials for ion pairs computed from the free-ion closedshell electron charge densities via the Gordon-Kim technique, ${ }^{5}$ this approach leads to accurate potential-energy surfaces for the systems under study.

However, when applied to study the phase transitions in $\mathrm{Rb}_{2} \mathrm{ZnCl}_{4},{ }^{6,7}$ this method become inadequate. It was found that the short-range $\mathrm{Zn}^{2+}-\mathrm{Cl}^{-}$potential for the $\mathrm{ZnCl}_{4}^{2-}$ ion computed from the electron charge densities of the free $\mathrm{Zn}^{2+}$ and $\mathrm{Cl}^{-}$ions was manifestly too hard and an overall reduction of this potential had to be adopted to give good agreement with the experimental $\mathrm{Zn}-\mathrm{Cl}$ bond length. Also, some distortions of the $\mathrm{ZnCl}_{4}^{2-}$ ion occurred, probably resulting from the combination of a lack of intramolecular bond-angle stiffness and full ionicity. This inadequacy of the method becomes even more obvious when it is used to treat $\mathrm{K}_{2} \mathrm{SeO}_{4}$, where the required closed-shell electroncharge-density distribution would give selenium the unrealistically large ionicity of $6+$.

The basic shortcoming of this approach is the failure to treat the molecular ions as single entities. We believe that this need is quite general for a variety of systems which contain molecular ions, e.g., $\mathrm{ZnCl}_{4}^{2-}, \mathrm{SeO}_{4}^{2-}$, $\mathrm{NO}_{2}^{-}, \mathrm{SO}_{3}^{-}$, etc. Therefore the starting point in our new approach is to perform ab initio quantum chemistry calculations for the whole molecular ion, say $\mathrm{SeO}_{4}^{2-}$ in the case of $\mathrm{K}_{2} \mathrm{SeO}_{4}$. The resultant electron-chargedensity distribution accounts for possible charge transfer between the atoms within the group and thus leads to realistic Gordon-Kim potentials for intermolecular interactions. As for the intramolecular interactions, for which the simple Gordon-Kim model becomes invalid due to covalency, we use Taylor expansions of the molecular ions' total energy which can be constructed from the quantum chemistry calculations. These expansions are adequate owing to the stiffness of the intramolecular bonds.

We applied our new approach to simulation of the phase transitions in $\mathrm{K}_{2} \mathrm{SeO}_{4}$, which, as the prototype of the series of the $A_{2} B X_{4}$ compounds, has attracted extensive experimental and theoretical efforts. ${ }^{8}$ At room temperature it has an orthorhombic paraelectric phase (space group Pnam ), ${ }^{9}$ with 4 formula units per unit cell, which changes to an incommensurate structure at 129.5 $\mathrm{K}\left(T_{i}\right)$. There follows a commensurate lock-in transition at $93 \mathrm{~K}\left(T_{c}\right)$ below which the crystal possesses an orthorhombic ferroelectric superstructure ${ }^{10}$ (space group $P n a 2_{1}$ ) whose a axis is triple that of the roomtemperature Pnam phase.

We first performed a quantum chemistry structural optimization for an isolated $\mathrm{SeO}_{4}^{2-}$ ion. Thus the calculation did not include a crystal-field background, since over $90 \%$ of the contribution to the electric fields at the selenium and oxygen sites are from the other ions in the same $\mathrm{SeO}_{4}^{2-}$ group. The optimized $\mathrm{SeO}_{4}^{2-}$ forms a perfect tetrahedron with the $\mathrm{Se}-\mathrm{O}$ bond length being 3.09 a.u., which is very close to the experimental average value of 3.07 a.u. in the Pnam phase. Then the force constants at this optimized structure are calculated to give a harmonic expansion for the total energy of this ion. Since throughout, in the Pnam, incommensurate, and $P n a 2_{1}$ phases, the $\mathrm{SeO}_{4}^{2-}$ ions stay as near-perfect tetrahedra, this expansion should give a satisfactory description of the Se-O and O-O interactions in the same $\mathrm{SeO}_{4}^{2-}$ ion.

We then calculated the electron charge density for the whole $\mathrm{SeO}_{4}^{2-}$ ion for the optimized structure and obtained charge densities for the individual $\mathrm{Se}$ and $\mathrm{O}$ atoms in the spirit of Mulliken population analysis. Pair potentials external to the $\mathrm{SeO}_{4}^{2-}$ units were then calculated via the Gordon-Kim technique using these charge densities and the free-ion charge density ${ }^{11}$ for $\mathrm{K}^{+}$. These intermolecular pair potentials are much easier to employ for crystal simulations than complicated molecule-molecule potentials and we are not restricted to treating the selenate ions as rigid rotors. The quantum chemistry calculations were performed using the GAUSSIAN 86, program ${ }^{12}$ and details of the calculations of the pair potentials will be given in future publications. ${ }^{13}$

With both the intramolecular and intermolecular interaction potentials determined we then performed a Pnam-symmetry-restricted static relaxation for $\mathrm{K}_{2} \mathrm{SeO}_{4}$, which should provide a rigorous test of theoretical 
TABLE I. Prototypic atomic positions for $\mathrm{K}_{2} \mathrm{SeO}_{4}$ in the relaxed Pnam structure. Experimental values (Ref. 9) are given in parentheses.

\begin{tabular}{ccccc}
\hline \hline Prototype & $x / a$ & $y / b$ & $z / c$ & $\begin{array}{c}\text { Wyckoff } \\
\text { designation }\end{array}$ \\
\hline $\mathrm{Se}$ & 0.2324 & 0.4203 & $\frac{1}{4}$ & $4(\mathrm{c})$ \\
$\mathrm{K}(1)$ & $(0.2242)$ & $(0.4200)$ & & \\
& 0.1589 & 0.0892 & $\frac{1}{4}$ & $4(\mathrm{c})$ \\
$\mathrm{K}(2)$ & $(0.1705)$ & $(0.0843)$ & & \\
& 0.9869 & 0.6927 & $\frac{1}{4}$ & $4(\mathrm{c})$ \\
$\mathrm{O}(1)$ & $(0.9943)$ & $(0.7095)$ & & \\
& 0.3074 & 0.3432 & 0.0183 & $8(\mathrm{~d})$ \\
$\mathrm{O}(2)$ & $(0.2931)$ & $(0.3471)$ & $(0.0271)$ & \\
& 0.3136 & 0.5728 & $\frac{1}{4}$ & $4(\mathrm{c})$ \\
$\mathrm{O}(3)$ & $(0.3024)$ & $(0.5644)$ & & \\
& 0.0101 & 0.4216 & $\frac{1}{4}$ & $4(\mathrm{c})$ \\
\hline \hline
\end{tabular}

potential-energy surface. The relaxation is done with a lattice potential-energy minimization program developed in our group. ${ }^{7,13}$

The lattice constants in the relaxed structure $(a=7.26$ $\AA, b=9.86 \AA, c=5.67 \AA$ ) have an overall shortening of about $6 \%$ from the experimental values ${ }^{9}(a=7.66 \AA$, $b=10.47 \AA, c=6.00 \AA)$, which is a rather general feature for large-unit-cell simulations using Gordon-Kim potentials. But the fractional basis vectors of the ions, as given in Table I, are very close to those in the experimental structure. The relaxed structure is plotted in Fig. 1(b), along with the experimental structure ${ }^{9}$ Fig. 1(a). For simplicity, only the projections of the ion positions parallel to the a axis are shown. As can be seen overall
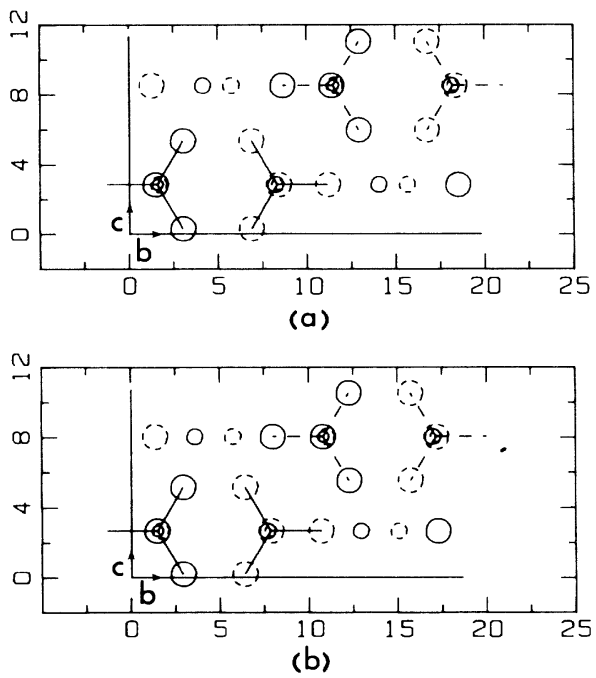

FIG. 1. Projections of ion positions parallel to the a axis in the (a) experimental and (b) simulated Pnam structure of $\mathrm{K}_{2} \mathrm{SeO}_{4}$. Bonds (straight lines) connect each selenium and the nearest four oxygen ions. The lattice constants are given in atomic units. the simulation agrees very well with the experimental data.

Next we performed a static relaxation for the lowertemperature $P$ na $2_{1}$ superstructure of $\mathrm{K}_{2} \mathrm{SeO}_{4}$, to provide a further test of the theoretical energy surfaces. In this phase the unit cell contains 12 formula units, in total 84 ions. Figure 2(a) gives the projections of the ion positions parallel the a axis in this phase, derived from experimental data at $80 \mathrm{~K} .{ }^{10}$ Comparing Fig. 1(a) with Fig. 2(a), we see that the Pna $2_{1}$ superstructure is the result of a modulation along the a axis involving shifts of the $\mathrm{K}^{+}$ions and relative rotations of the $\mathrm{SeO}_{4}^{2-}$ ions, which are aligned in the Pnam structure. Figure 2(b) gives the structure obtained by static relaxation with the Pna $2_{1}$-symmetry restriction. Apart from a shortening of the lattice constants similar to that in the Pnam phase, the relaxation again gives a close fit to the experimental structure. The basic modulation in the experimental structure is closely simulated, although the amplitudes are slightly different.

Given these excellent agreements with the experimental structure, we performed a nonconstrained moleculardynamics (MD) "quench" for a $3 \mathbf{a}$ by $2 \mathbf{b}$ by 2 c supercell containing 336 ions, using a constant (zero) pressure molecular-dynamics program also developed in our group. $^{7,13}$ Starting from the Pnam structure, the sample was first sufficiently relaxed to be as free of experimental information as possible and then cooled down in stages to $T=0 \mathrm{~K}$. We found that we could indeed reproduce the observed Pnam-Pna $2_{1}$ phase transition by cooling in stages allowing an equilibration time of 2 ps at each
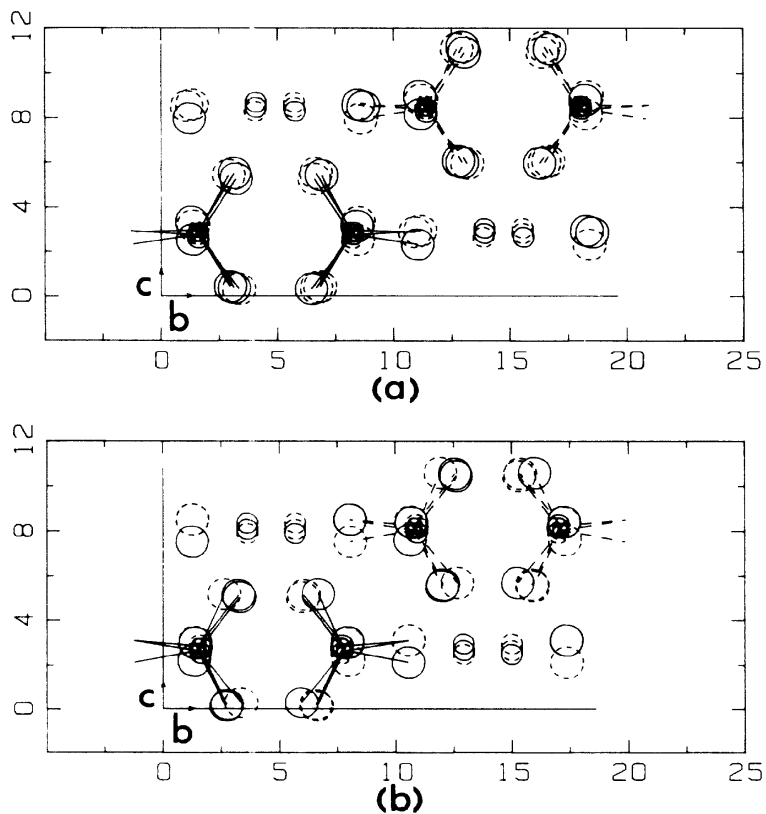

FIG. 2. Projections of ion positions parallel to the a axis in the (a) experimental and (b) simulated Pna 2 , superstructure of $\mathrm{K}_{2} \mathrm{SeO}_{4}$. 
stage. Figure 3 shows the b-c cross sections of the simulated structures at (a) $T=300 \mathrm{~K}$ and (b) $T=57 \mathrm{~K}$, both averaged over $2 \mathrm{ps}$. In this figure, the average $\mathrm{Se}-\mathrm{O}$ bonds are indicated by straight lines and the thermal motions of the ions are given by ellipsoids centered at the average ion positions. The structure in Fig. 3(a) is virtually the same as the experimental Pnam structure. The large (about 1 bohr) amplitudes of the thermal motions of some of the oxygen ions indicate that the crystal at this temperature is actually in a dynamically disordered state. This is in accord with a previous hypothesis made on the basis of the internal-mode Raman spectra. ${ }^{14}$ The structure in Fig. 3(b) is clearly the Pna $2_{1}$ structure given in Fig. 2(a). The positions of some of the ions appear slightly different, but again the basic modulation of the ions along the a axis agrees well with the experimental structure. In fact, when further cooled to $T=0 \mathrm{~K}$, this structure agrees closely with the theoretical Pna $2_{1}$ structure [Fig. 2(b)] both in structure and in potential energy.

In order to monitor more precisely the phase transition, we performed a CPU-intensive MD calculation for a 3 a by 2 b by $4 c$ supercell ( 672 ions) with 40 -ps averaging time at each temperature, and then calculated the rms static deviation $R$ of the Se-O bonds from their orientations in the Pnam phase as a convenient order parameter. The result is plotted in Fig. 4 together with the value of $R$ for the experimental Pna $2_{1}$ structure at 80

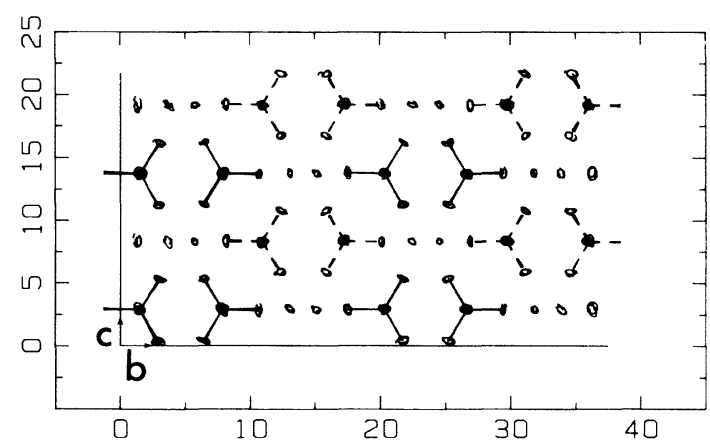

(a)

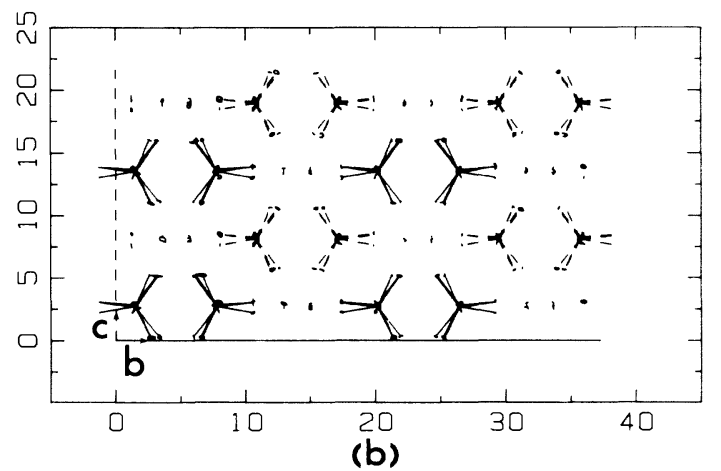

FIG. 3. Projections of the average ion positions in the structures at (a) $T=300 \mathrm{~K}$ and (b) $T=57 \mathrm{~K}$ obtained in the dynamics simulation for $\mathrm{K}_{2} \mathrm{SeO}_{4}$. The ellipsoids indicate the thermal motions of the ions.
K. ${ }^{9}$ Figure 4 shows that the onset of the transition lies in the range $120-140 \mathrm{~K}$, which corresponds closely to the incommensurate phase transition at $T_{i}=129.5 \mathrm{~K}$, and the transition is virtually complete at about the ferroelectric lock-in transition $T_{c}=93 \mathrm{~K}$. The behavior of the order parameter around $200 \mathrm{~K}$ was observed in several simulations; it is probably a dynamical, but slow, precursor to the main transition. To clarify this point would require a more extensive computation for an even larger number of ions, and this is beyond our computing resources.

The above excellent agreement with the experimental data leads us to conclude that we indeed have found a very realistic $a b$ initio potential-energy surface of $\mathrm{K}_{2} \mathrm{SeO}_{4}$ and from it we have obtained a first-principles explanation for the driving mechanism of the phase transitions in $\mathrm{K}_{2} \mathrm{SeO}_{4}$. From Fig. 2(b) we see that the modulation that changes the Pnam phase to the Pna $2_{1}$ superstructure is characterized by rotations of the selenate ions in two opposite directions away from their orientations in the Pnam structure. This suggests that the two rotated positions of the selenate ions may be equivalent in energy. This is indeed the case, as we found by explicit calculation for a single unit cell of 28 ions; both are $12.3 \mathrm{meV}$ per formula unit lower than the Pnam structure. Since the Pna $2_{1}$ structure is $10.7 \mathrm{meV}$ per formula unit lower in energy than the Pnam phase, these two structures are effectively degenerate for temperatures $\sim 100 \mathrm{~K}$ and above. As a consequence when $k T$ becomes less than $\sim 10-15 \mathrm{meV}$, and the structure transforms, the state selected will be that of higher entropy. Clearly, this is the Pna $2_{1}$ phase in which, for example, the $\mathrm{SeO}_{4}^{2-}$ ions have three rather than one possible orientation. Furthermore, for the Pna 21 structure to transform to the single-cell, selenate-rotated, phase requires that at least one of the tetrahedra has to switch through the Pnam configuration. As the system is further cooled this becomes increasingly difficult, and when $k T \sim 1-2 \mathrm{meV}$ (when the single cell phase would become preferred energetically), the transformation is frustrated by the $\sim 10-\mathrm{meV}$ activation barrier, and the Pna $2_{1}$

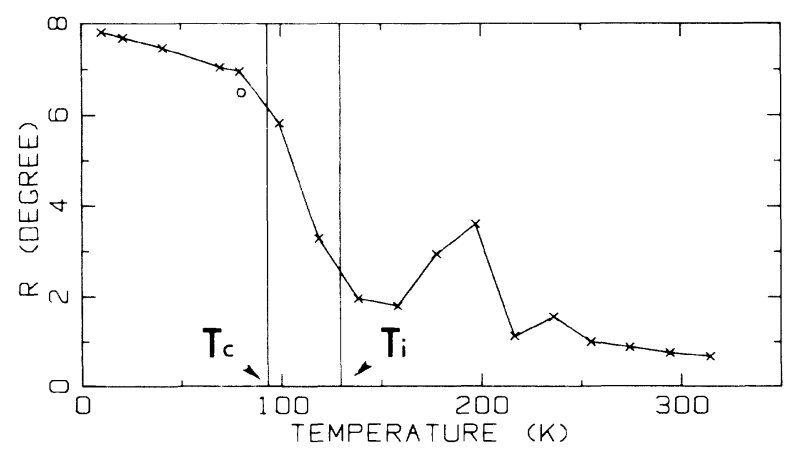

FIG. 4. Theoretical ( $x$ ) and experimental (o) orderparameter values as functions of temperature. 
phase persists.

This work was supported by the U.S. Army Research Office. We should also like to acknowledge many helpful discussions with Dr. P. J. Edwardson and Dr. D. P. Billesbach.

'L. L. Boyer, Phys. Rev. Lett. 42, 584 (1979). A fuller account is given in Phys. Rev. B 23, 3673 (1981).

${ }^{2}$ L. L. Boyer, Phys. Rev. Lett. 45, 1858 (1980); 46, 1172 (1981).

${ }^{3}$ L. L. Boyer and J. R. Hardy, Phys. Rev. B 24, 2527 (1981).

${ }^{4}$ L. L. Boyer, J. Phys. C 17, 1825 (1984).

${ }^{5}$ R. G. Gordon and Y. S. Kim, J. Chem. Phys. 56, 3122 (1971).
${ }^{6}$ V. Katkanant, P. J. Edwardson, J. R. Hardy, and L. L. Boyer, Phys. Rev. Lett. 57, 2033 (1986).

${ }^{7}$ P. J. Edwardson, V. Katkanant, J. R. Hardy, and L. L. Boyer, Phys. Rev. B 35, 8470 (1987).

${ }^{8}$ See M. S. Haque and J. R. Hardy, Phys. Rev. B 21, 245 (1980), and references therein.

${ }^{9}$ A. Kalman, J. S. Stephens, and D. W. J. Cruickshank, Acta Crystallogr. Sect. B 26, 1451 (1970).

${ }^{10}$ N. Yamada, Y. Ono, and T. Ikeda, J. Phys. Soc. Jpn. 53, 2565 (1984).

${ }^{11 E}$ E. Clementi and C. Roetti, At. Data Nucl. Data Tables 14, 177 (1974).

${ }^{12}$ M. J. Frisch et al., GAUSSIAN 86 (Carnegie-Mellon Quantum Chemistry Publishing Unit, Pittsburgh, PA, 1984).

${ }^{13}$ H. M. Lu and J. R. Hardy (to be published).

${ }^{14}$ V. Katkanant, J. R. Hardy, and F. G. Ullman, Ferroelectrics 82, 185 (1988). 